\title{
Poly(oxyethylene) Based Surface Coatings for Poly(dimethylsiloxane) Microchannels
}

\author{
Wibke Hellmich, Jan Regtmeier, Thanh Tu Duong, Robert Ros, \\ Dario Anselmetti, and Alexandra Ros* \\ Experimental Biophysics and Applied Nanosciences, Physics Faculty, \\ Bielefeld University, Germany
}

Received April 19, 2005. In Final Form: May 31, 2005

\begin{abstract}
Control of surface properties in microfluidic systems is an indispensable prerequisite for successful bioanalytical applications. Poly(dimethylsiloxane) (PDMS) microfluidic devices are hampered from unwanted adsorption of biomolecules and lack of methods to control electroosmotic flow (EOF). In this paper, we propose different strategies to coat PDMS surfaces with poly(oxyethylene) (POE) molecules of varying chain lengths. The native PDMS surface is pretreated by exposure to UV irradiation or to an oxygen plasma, and the covalent linkage of $\mathrm{POE}$-silanes as well as physical adsorption of a triblock-copolymer (F108) are studied. Contact angle measurements and atomic force microscopy (AFM) imaging revealed homogeneous attachment of POE-silanes and F108 to the PDMS surfaces. In the case of F108, different adsorption mechanisms to hydrophilic and hydrophobic PDMS are discussed. Determination of the electroosmotic mobilities of these coatings in PDMS microchannels prove their use for electrokinetic applications in which EOF reduction is inevitable and protein adsorption has to be suppressed.
\end{abstract}

\section{Introduction}

In recent years, PDMS has been intensively used as a fabrication material for microdevices in many fields of application due to its relatively low fabrication costs, moderate clean room requirements, and ease of assembly. Its remarkable gas permeability and biocompatibility have in particular attracted the integration of bioanalytical applications into PDMS devices. A recent report by Sia and Whitesides ${ }^{1}$ reviews bioanalytical applications comprising immunoassays, separation of biomolecules, as well as sorting and manipulation of cells.

Electrophoretic separations of biological compounds in microfluidic devices strongly depend on the properties of the microchannel surface material. For DNA applications, the PDMS surface properties are mostly appropriate so that separations of DNA samples ${ }^{2-5}$ and chromosome isolation ${ }^{6}$ have been reported. In contrast, for proteins and peptides, PDMS is considered as a critical material due to its hydrophobic nature favoring adsorption of apolar molecules. ${ }^{7-8}$ Furthermore, electrokinetic properties of PDMS devices have to be controlled in order to obtain competitive separation results.

Electrokinetic transport of analytes in microchip capillary electrophoresis is dominated by the electrophoretic transport of the charged analytes and the liquid transport caused by electroosmosis within these channels. On a charged channel surface, electroosmotic flow (EOF) occurs

\footnotetext{
* To whom correspondence should be addressed.

(1) Sia, S. K.; Whitesides, G. M. Electrophoresis 2003, 24, 3563-676.

(2) Effenhauser, C. S.; Bruin, G. J. M.; Paulus, A.; Ehrat, M. Anal. Chem. 1997, 69, 3451-57.

(3) Inatomi, K.; Izuo, S.; Lee, S.; Ohji, H.; Shiono, S. Microelectron. Eng. 2003, 70, 13-18.

(4) Kaji, N.; Tezuka, Y.; Takamura, Y.; Ueda, M.; Nichimoto, T.; Nakanishi, H.; Horiike, Y.; Baba, Y. Anal. Chem. 2004, 76, 15-22.

(5) Ros, A.; Hellmich, W.; Duong, T.; Anselmetti, D. J. Biotechnol. 2004, 122, 65-67.

(6) Prinz, C.; Tegenfeldt, J. O.; Austin, R. H.; Cox, E. C.; Sturm, J. C. Lab Chip 2002, 2, 207-11.

(7) Linder, V.; Verpoorte, E.; Thormann, W.; de Rooij, N.; Sigrist, H. Anal. Chem. 2001, 73, 4181-89.

(8) Ocvirk, G.; Munroe, M.; Tang, T.; Oleschuk, R.; Westra, K.; Harrison, D. J. Electrophoresis 2000, 21, 107-15.
}

by the application of an electric field, since counterions form a diffusive layer which are drawn toward an electrode. Shear forces in the liquid lead to a unique flow within the whole capillary with electroosmotic velocity $v_{\text {eo }}$, defined by the Schmoluchowsky equation

$$
v_{\text {eо }}=\frac{E \epsilon \zeta}{\eta}
$$

where $E, \epsilon, \eta$, and $\zeta$ denote the applied electric field, the permittivity of the solution, the viscosity of the solution, and the zeta potential of the surface. The electroosmotic mobility $\mu_{\mathrm{e}}$ is defined as the electroosmotic velocity per unit field strength $\mu_{\mathrm{eo}}=\epsilon \xi / \eta(2)$. The surface charge density, $\delta$, is related to the zeta potential via $\delta=\epsilon \kappa \zeta$ (3) with $\kappa^{-1}$ the thickness of the electrical double layer (Debye thickness). The measurement of the electroosmotic mobility in a capillary thus allows the determination of the zeta potential and the surface charge density. From eqs 2 and 3 , it follows that EOF can be controlled by an adequate choice of the buffer composition, the thickness of the double layer, the control of surface charges through adequate coating, and the viscosity of the solution.

Microchannels composed of PDMS exhibit moderate EOF which can be enhanced by oxidative treatments increasing the amount of dissociable groups leading to negative charges on the surface. It is commonly accepted that these treatments enhance the amount of $\mathrm{Si}-\mathrm{OH}$ groups on the surface which could be demonstrated by infrared spectroscopy ${ }^{9}$ and X-ray photoelectron sprectroscopy. ${ }^{10-11}$

In conventional capillary electrophoresis, coating strategies to control EOF have been studied extensively. ${ }^{12}$ Among them, POE derivatives have been demonstrated

(9) Ren, X.; Bachman, M.; Sims, C.; Li, G. P.; Allbritton, N. J. Chromatogr. B 2001, 762, 117-25.

(10) Efimenko, K.; Wallace, W. E.; Genzer, J. J. Colloid Interface Sci. 2002, 254, 306-15.

(11) Owen, M. J.; Smith, P. J. J. Adhes. Sci. Technol. 1994, 8, 106375.

(12) Beale, S. C. Anal. Chem. 1998, 70, 279-300. 
to significantly reduce EOF, simultaneously diminish protein adsorption, and therefore provide good separations of proteins ${ }^{13}$ with an increase in separation efficiency. ${ }^{14}$

On PDMS microchips, several coating strategies have been proposed to enhance separation performance through chemical surface engineering. Most of them have been summarized in a recent review. ${ }^{15}$ Dynamic coating strategies using surfactant molecules ${ }^{16}$ as well as polyelectrolyte multilayers ${ }^{17}$ have been employed to enhance the separation efficiency and control EOF. Reactive polymer coatings have also been suggested to provide control over electrokinetic properties in PDMS devices. ${ }^{18}$ Furthermore, aminoterminated silanes could be covalently coupled to PDMS ${ }^{19-20}$ where electroosmotic flow reversal could be demonstrated.$^{20} \mathrm{~A}$ method, based on a silanisation strategy of poly (oxyethylene) (POE) derivatives on PDMS has been suggested by Delamarche and co-workers ${ }^{21-23}$ for applications in micro contact printing. However, the electrokinetic impact of these coatings on PDMS microdevices has not been reported yet. More recently, photo initiated grafting has been shown to be able to form grafted poly(ethylene oxide) on PDMS channels. ${ }^{24}$

In this paper, we focus on the coating of POEs to PDMS microchannel surfaces addressing two coating strategies in aqueous solution: first, a covalent attachment of alkoxysilane POEs to hydrophilic, silanol groups containing PDMS surfaces and, second, adsorption of a Pluronic triblock copolymer compound to either hydrophobic (native) or hydrophilic (oxidized) PDMS surfaces. The coated surfaces are characterized by contact angle measurements and AFM and the impact for electrophoretic applications is analyzed by EOF measurements.

\section{Materials and Methods}

Chemicals and Reagents. PDMS (Sylgard 184) was purchased from Dow Corning (USA). Glass slides $(76 \mathrm{~mm} \times 26 \mathrm{~mm}$ ) were from Menzel (Germany), Si wafers (P-Type 100, doped with boron) from CrysTec (Germany), and the 1000 square mesh grids (G 2780N) from AGAR (Germany). 2-[Methoxy(polyethyleneoxy)propyl] trimethoxysilane, $\mathrm{MW} \sim 460,\left(\mathrm{Si}-\mathrm{POE}_{(8)}, 6\right.$ to $9 \mathrm{POE}$ units in accordance with the manufacturer, see also Table 1) and tridecafluoro-1,1,2,2-tetrahdyrooctyl-1-trichlorsilane (TTTS) were obtained from ABCR (Germany). Poly(ethyleneoxy)di(triethoxy)silane, $\mathrm{MW} \sim 3400,\left(\mathrm{Si}-\mathrm{POE}_{(70)}-\mathrm{Si}\right.$, see Table 1) and methoxy(poly(ethyleneoxy))triethoxysilane, $\mathrm{MW} \sim 5000,\left(\mathrm{Si}-\mathrm{POE}_{(109)}\right.$, see Table 1) were from Nektar (USA). Polyethyleneoxy (POE)polyoxypropylene (POP)-POE triblock copolymers Pluronic F108 (MW 14600) and L101 (MW 3800) were donated by BASF (Germany), and disodium hydrogen phosphate dihydrate was

(13) Maegher, R. J.; Seong, J.; Laibinis, P. E.; Barron, A. E. Electrophoresis 2004, 25, 405-14.

(14) Ng, C. L.; Lee, H. K.; Li, S. F. Y. J. Chromatogr. A 1994, 659, 427-34

(15) Makamba, H.; Kim, J. H.; Lim, K.; Park, N.; Hahn, J. H. Electrophoresis 2003, 24, 3607-19.

(16) Badal, M.Y.; Wong, M.; Chiem, N.; Salimi-Moosavi, H.; Harrison, D. J. J. Chromatogr. A 2002, 947, 277-86.

(17) Liu, Y.; Fanguy, J. C.; Bledsoe, J. M.; Henry, C. S. Anal. Chem. 2002, 72, 5939-44.

(18) Lahann, J.; Balcells, M.; Lu, H.; Rodon, T.; Jensen, K. F.; Langer, R. Anal. Chem. 2003, 75, 2117-22. 11 .

(19) Diaz-Quijada, G. A.; Wayner, D. M. Langmuir 2004, 20, 9607-

(20) Wang, B.; Chen, L.; Abdulali-Kanji, Z.; Horton, J. H.; Oleschuk, R. D. Langmuir 2003, 19, 9792-98.

(21) Donzel, C.; Geissler, M.; Bernard, A.; Wolf, H.; Michel, B.; Hilborn, J.; Delamarche, E. Adv. Mater. 2001, 15, 1164-67.

(22) Papra, A.; Bernard, A.; Juncker, D.; Larsen, N. B.; Michel, B.; Delamarche, E. Langmuir 2001, 17, 4090-95.

(23) Delamarche, E.; Donzel, C.; Kamounah, F. K.; Wolf, H.; Geissler, M.; Stutz, R.; Schmidt-Winkel, P.; Michel, B.; Mathieu, H. J.; Schaumburg, K. Langmuir 2003, 19, 8749-58.

(24) Hu, S.; Ren, X.; Bachman, M.; Sims, C. E.; Li, G. P.; Allbritton, N. L. Anal. Chem. 2004, 76, 1865-70.
Table 1. Polymer Chain Length of Used Compounds and Derivatization Mechanism

\begin{tabular}{lccl}
\hline $\begin{array}{c}\text { derivatization } \\
\text { agent }\end{array}$ & $\begin{array}{c}\text { number of } \\
\text { POE units } \\
\text { in polymer } \\
\text { branch }\end{array}$ & $\begin{array}{c}\text { number of } \\
\text { POP units } \\
\text { in polymer } \\
\text { branch }\end{array}$ & $\begin{array}{c}\text { PDMS surface } \\
\text { attachment via }\end{array}$ \\
\hline $\mathrm{Si}_{-\mathrm{POE}_{(8)}{ }^{a}}$ & $6-9$ & & silanol groups \\
$\mathrm{Si}_{-} \mathrm{POE}_{(70)-} \mathrm{Si}^{b}$ & 70 & & silanol groups \\
$\mathrm{Si}-\mathrm{POE}_{(109)}{ }^{a}$ & 109 & 52 & silanol groups \\
$\mathrm{F} 108$ & $132^{c}$ & 52 & adsorption \\
$\mathrm{L} 101$ & $9^{c}$ & 52 adsption
\end{tabular}

${ }^{a}$ Monofunctional, terminal silane. ${ }^{b}$ Bifunctional, terminal silane. ${ }^{c}$ Two POE branches per molecule.

from Fluka (Germany). SU-8 (50) negative photoresist, thinner $\gamma$-butyrol acetone, and developer propyleneglycolmethyl ether acetate were obtained from Microresist (Germany). Deionized water was supplied from a Milli-Q biocel purification unit (Millipore,USA).

Fabrication of PDMS Devices. The fabrication of the microchip can be divided into two parts: (i) fabrication of a master wafer with the desired microstructures for multiple PDMS replica castings and (ii) the production of the PDMS replica itself. The fabrication procedure for the master wafer with the inverted structures was recently published. ${ }^{25}$ Briefly, a Si wafer was spin coated with a negative photoresist SU-8, UV-exposed through a chromium mask, and developed in a developer bath. Additional silanization of the master wafer in a vacuum exsiccator for 30 min with TTTS enabled multiple usage of the same wafer for PDMS replica casts. A mixture of Sylgard 184 and its curing agent in a ratio of 10:1 was poured over the wafer. After curing at a temperature of $85^{\circ} \mathrm{C}$ for $4.5 \mathrm{~h}$, the cross-linked polymer was easily peeled off the wafer. With this method, PDMS channels with a width of $20 \mu \mathrm{m}$ and a depth of $20 \mu \mathrm{m}$ were formed on a corresponding master wafer. Reservoir holes were punched through the structured side for fluid access. The structured PDMS slab was then covered with an unstructured PDMS slab or a clean glass slide. Before assembly, the PDMS channels and cover slides were either left untreated, UV treated, or exposed to an oxygen plasma. Directly after assembly, the microchannels were initially filled with a $20 \mathrm{mM}$ phosphate buffer at $\mathrm{pH} 8.2$.

Flat PDMS surfaces were created by casting PDMS on an unstructured silanized wafer for the contact angle and AFM measurements.

PDMS Surface Oxidation. The PDMS surfaces were either treated by UV light in ambient atmosphere or by an oxygen plasma. For the UV treatment, PDMS slabs were placed in an UVO cleaner (model 42-220, Jelight, USA) for 3 or 60 min under ambient atmosphere. For oxygen plasma modification, samples were treated in a home-built glow discharge unit in accordance with Aebi et al. ${ }^{26}$ In contrast to their work, we used a vacuum chamber creating controlled and defined oxygen atmospheres. Samples were placed on a grounded aluminum plate, and the chamber was evacuated to a pressure of $10^{-6}$ mbar before an oxygen atmosphere of 0.1 mbar was created. The tesla coil (BD$10 \mathrm{ASV}$ Electro-Technic Products Inc. (USA)) operating at $50 \mathrm{kV}$ at $500 \mathrm{kHz}$ is attached to the second parallel electrode, separated by $6.2 \mathrm{~cm}$ from the aluminum electrode, providing high voltage glow discharges.

Derivatization of PDMS Surfaces. Native PDMS or PDMS slabs were coated with three POE silanes immediately after oxidative treatment in a $3 \mathrm{mM}$ solution in water with $0.8 \mathrm{~mL}$ of concentrated $\mathrm{HCl}$ per liter for $2 \mathrm{~h}$. Adsorption of Pluronics was performed in a $3 \mu \mathrm{M}$ solution in $10 \mathrm{mM}$ phosphate buffer ( $\mathrm{pH} 8.2$ ) for $20 \mathrm{~h}$. After the incubation, the samples were thoroughly washed in water and dried in a stream of $\mathrm{N}_{2}$.

Contact Angle Measurements. A contact angle goniometer (Krüss system G10, Hamburg, Germany) was used to determine the wettability of the modified PDMS surfaces by water. On each sample the advancing contact angle was measured at several spots. The resulting values were averaged with an absolute error of $2^{\circ}$.

(25) Duong, T.; Kim, G.; Ros, R.; Streek, M.; Schmid, F.; Brugger, J.; Ros, A.; Anselmetti, D. Microelectron. Eng. 2003, 67-68, 905-12. (26) Aebi, U.; Pollard, T. D. J. Electron Microsc. Tech. 1987, 33. 
Surface Characterization using AFM. For coating thickness monitoring by AFM, a 1000 square mesh grid was placed onto the PDMS slab. After plasma treatment for $30 \mathrm{~s}$, the grid was removed and the PDMS slab was either directly examined or immersed into a $3 \mu \mathrm{M} \mathrm{F} 108$ solution in $10 \mathrm{mM}$ phosphate buffer $\mathrm{pH} 8.2$ for $20 \mathrm{~h}$. Then the slab was thoroughly washed, and a phosphate buffer drop (10 mM, pH 8.2) was placed onto it. AFM measurements were performed with a commercial instrument (Bioscope, Nanoscope IIIa, Veeco, USA). Images under ambient conditions were acquired with silicon cantilevers (Nanoprobe, Wetzlar, Germany) in tapping mode of operation. For in situ AFM measurements in solution (10 mM phosphate buffer $\mathrm{pH}$ 8.2), standard silicon nitride cantilevers (Olympus, Japan) were used. The roughness of the surfaces topographies was characterized by measuring the root-mean-square (rms) roughness.

EOF Measurements. The current monitoring method according to Huang et al. ${ }^{27}$ was used to measure $\mu_{\mathrm{e}}$ in the microchannels. An electrical field $E$ was applied to the microchannels across two platinum electrodes immersed into each reservoir. Electrodes were connected to power supplies from FUG (model HCN 14-12500 and HCN 7E-12500, Germany) via a relay circuit. Electric field and current were controlled and recorded via a labview program (National Instruments, USA).

A stable current was established with the $20 \mathrm{mM}$ phosphate buffer initially filled into the microchannels. In one reservoir, the $20 \mathrm{mM}$ buffer was then replaced by an $18 \mathrm{mM}$ buffer. After monitoring the current decline, the microchannels were washed several times with $20 \mathrm{mM}$ buffer. The electroosmotic mobility $\mu_{\mathrm{eo}}$ was calculated using $\mu_{\mathrm{eo}}=L /(E t)$, where $L$ is the channel length. The time, $t$, for complete buffer exchange in the microchannel was computed by calculating the point of interception of two linear fits applied to the monitored data. The interchannel variance of this method applied to PDMS devices has been reported to be as high as $30 \%{ }^{9}$ and was confirmed in our studies, whereas the standard deviation from the same device is smaller than $10 \%$. Thus, for comparative studies of EOF reduction through POE coatings, reference measurements for each channel were carried out before derivatization, and EOF reduction was measured directly after surface modification and after specific periods of time. The resulting reduction of $\mu_{\mathrm{eo}}$ was determined relative to its initial value.

\section{Results and Discussion}

In the following, we will present and discuss the coating of POE derivatives to PDMS surfaces by means of adsorption and covalent attachment. PDMS treated with reactive oxygen is described in the literature as resembling glass surfaces in their chemical properties due to the creation of silanol groups on the surface which support cathodic EOF. ${ }^{28}$ Hence, for the covalent attachment, alkoxysilanes have been chosen and oxidative treatments of PDMS to enhance hydrophilicity and thus the silanol content were investigated. Furthermore, PDMS coating through adsorption is explored with a triblock copolymer capable of adsorbing on hydrophilic and hydrophobic PDMS surfaces.

Native and Treated PDMS. PDMS surfaces were either treated by an oxygen plasma or by UV light in ambient atmosphere. Table 2 demonstrates the resulting contact angles by these treatments. UV treatment in ambient atmosphere for 3 min reduces the contact angle of native PDMS $\left(117^{\circ}\right)$ only slightly to $115^{\circ}$. Treatments longer than 30 min significantly enhance the hydrophilicity of the PDMS surface, but since cracks occur in the PDMS, these surfaces are not suitable for microfluidic experiments. Efimenko and co-workers ${ }^{10}$ have found that the material density within a $5 \mathrm{~nm}$ layer on PDMS treated

(27) Huang, X.; Gordon, M. J.; Zare, R. N. Anal. Chem. 1988, 60, $1837-38$

(28) Duffy, D. C.; Cooper McDonald, J.; Schueller, O. J. A.; Whitesides, G. M. Anal. Chem. 1998, 70, 4974-84.
Table 2. Contact Angles of PDMS Surfaces and $\mu_{\mathrm{eo}}$ of PDMS Microchannels before and after Oxidative Treatment

\begin{tabular}{clcl}
\hline device & $\begin{array}{c}\text { PDMS surface } \\
\text { treatment }\end{array}$ & $\begin{array}{c}\text { contact angle } \\
\text { of PDMS surface } \\
\text { Q }\left[^{\circ}\right]\end{array}$ & $\begin{array}{c}\mu_{\mathrm{eo}} \times 10^{-4} \\
{\left[\mathrm{~cm}^{2} / \mathrm{Vs}\right]^{a}}\end{array}$ \\
\hline PDMS/glass & native & 117 & $2.3(0.04)$ \\
& UV (3 min) & 115 & $2.6(0.1)$ \\
& UV (60 min) & 25 & n.a. \\
& plasma $(30 \mathrm{~s})$ & $<10$ & $3.1(0.5)$ \\
PDMS/PDMS & nlasma $(60 \mathrm{~s})$ & $<10$ & - \\
& native & & $1.7(0.1)$ \\
& UV (3 min) & & $3.1(0.3)$ \\
& plasma $(30 \mathrm{~s})$ & & $3.4(0.3)$
\end{tabular}

${ }^{a}$ Standard deviations are given in brackets.

by UV irradiation in oxygen containing atmosphere reached $\sim 50 \%$ that of silica. Furthermore, cracks on PDMS surfaces after oxygen plasma treatment have been reported to be due to the creation of a thin and brittle silicalike $\left(\mathrm{SiO}_{x}\right)$ layer. ${ }^{11,21,29-31}$ It is likely that the creation of cracks on prolonged UV-treated PDMS has its origin in the creation of a silica layer. On the other hand, PDMS treated with an oxygen plasma for 30 or $60 \mathrm{~s}$ yielded minimized contact angles smaller than $10^{\circ}$ and a hydrophilic surface in a very short time. Troublesome cracks were apparent after oxygen plasma treatment $\geq 60 \mathrm{~s}$, so that $30 \mathrm{~s}$ were taken for all other experiments.

The electrophoretic mobilities of treated PDMS channels reflected the trend observed by contact angle measurements (see Table 2). The values for $\mu_{e o}$ increased from native over UV-treated to plasma-treated PDMS for both the PDMS/PDMS and hybrid PDMS/glass microchannels. This is an indication that additional silanol-groups were created on the plasma-treated PDMS, which deprotonate in the employed buffer at $\mathrm{pH} 8.2$ and increase the negatively charged silanol groups on the surface. Additional negative charges enhance the zeta potential and thus lead to elevated EOF in plasma-treated channels. Our values for $\mu_{\mathrm{eo}}$ deviate slightly from a previous study, in which mobilities of 1.8 (native) and $3.6 \times 10^{-4} \mathrm{~cm}^{2} / \mathrm{Vs}$ (plasma-treated) are reported for $20 \mathrm{mM}$ phosphate buffer at pH 7.0 in semispherical PDMS channels with a PDMS to glass ratio of 1.5. ${ }^{9}$ This could be due to different buffer conditions and to an enhanced PDMS to glass ratio of 3 employed in our studies for the hybrid microchannels.

Oxidized PDMS and glass are both well-known for supporting EOF ${ }^{28}$ a fact which we have underlined with our EOF measurements of PDMS/PDMS as well as hybrid PDMS/glass microchannels (see Table 2). Therefore, for ease of use, all EOF measurements presented below were based on PDMS/glass composite channels with the premise of similar surface chemistries on oxidized PDMS and on glass surfaces.

Covalent Attachment of POE. For the covalent attachment of $\mathrm{POE}$ on PDMS, we have further investigated a method described by Delamarche et al. in which alkoxysilanes with varying lengths of POE chains are coupled to the oxidized PDMS surface containing silanol groups. ${ }^{21-23}$ Table 3 shows the contact angles resulting from the derivatization with $\mathrm{Si}-\mathrm{POE}_{(8)}, \mathrm{Si}-\mathrm{POE}_{(70)}-\mathrm{Si}$, and $\mathrm{Si}-\mathrm{POE}_{(109)}$ on PDMS surfaces with previous UV and plasma treatment. As expected, the contact angles are only reduced by the attachment of silanes to maximal $6 \%$

(29) Hillborg, H.; Ankner, J. F.; Gedde, U. W.; Smith, G. D.; Yasuda, H. K. Wikström, K. Polymer 2004, 41, 6851-63.

(30) Kim, J.; Chaudhury, M. K.; Owen, M. J. J. Colloid Interface Sci. 2000, 226, 231-36.

(31) Hillborg, H.; Tomaczak, N.; Olah, A.; Schönherr, H.; Vansco, G. J. Langmuir 2004, 20, 785-94. 


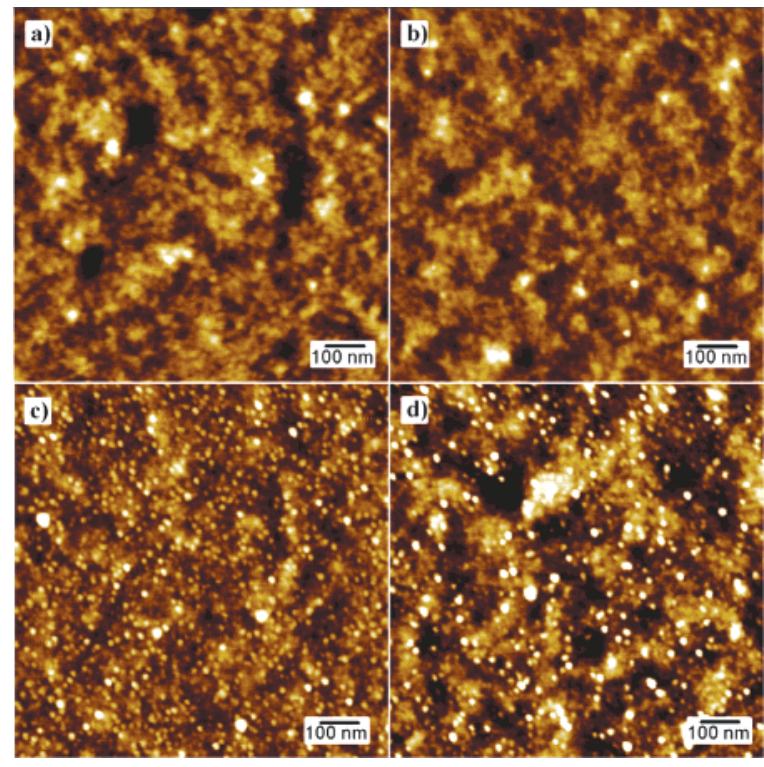

Figure 1. AFM images under ambient conditions of (a) an oxidized PDMS surface in comparison to (b) $\mathrm{Si}-\mathrm{POE}_{(8)}$, (c) $\mathrm{Si}-$ $\mathrm{POE}_{(70)}-\mathrm{Si}$, and (d) $\mathrm{Si}-\mathrm{POE}_{(109)}$ treated surfaces. The $z$ scale is from 0 to $5 \mathrm{~nm}$.

Table 3. Contact Angles of PDMS after POE-Coating with Different Pretreatments

\begin{tabular}{lccc}
\hline derivatization agent & native & UVO & plasma \\
\hline $\mathrm{Si}-\mathrm{POE}_{(8)}$ & 116 & 113 & 23 \\
$\mathrm{Si}-\mathrm{POE}_{(70)-\mathrm{Si}}$ & 111 & 108 & 47 \\
$\mathrm{Si}-\mathrm{POE}(109)$ & 113 & 112 & 46 \\
$\mathrm{~F} 108$ & 112 & 112 & 53 \\
L101 & 114 & 111 & 31
\end{tabular}

for UV-treated PDMS. In previous work, ${ }^{10}$ the existence of hydroxyl groups on the surface of UV-treated PDMS surfaces was reported. However, our data suggest a much lower fraction of silanol groups on the UV-treated compared to the plasma-treated PDMS (see also contact angles, Table 2). Control experiments with the silane substrates on native PDMS were also performed which showed practically no change of contact angle after silane treatment (see Table 3) confirming that covalent surface linkage due to the lack of silanol groups was not possible. Increased attachment of the POE-silanes to the plasmatreated PDMS surface is confirmed by an increase in contact angle after the silanization step. The obtained advancing contact angles of $\sim 50^{\circ}$ for $\mathrm{Si}-\mathrm{POE}_{(70)}-\mathrm{Si}$ and $\mathrm{Si}-\mathrm{POE}_{(109)}$ are in reasonable agreement with the advancing contact angles of $\sim 55^{\circ}$ reported by Papra et al. ${ }^{22}$ for POE-silanes on PDMS. However, the silane with the shortest POE units exhibits the smallest contact angle $\left(23^{\circ}\right)$ and represents thus the most hydrophilic surface.

Contact angle measurements together with the observed mobility in PDMS channels lead us to the conclusion that PDMS surfaces treated with oxygen plasma are best suited for the covalent attachment of alkoxysilanes to PDMS surfaces. For that reason, only plasma-treated surfaces were further studied for the covalent attachment of POEsilanes.

AFM investigations were carried out in order to elucidate the topography change and the coating homogeneity of silanes on the PDMS surface. Figure 1 demonstrates a series of AFM images of silanized PDMS. In the case of the shortest POE-silane the topography after attachment to the oxidized PDMS surface has not been changed significantly. However, the rms roughness decreased from $0.55 \mathrm{~nm}$ for oxidized PDMS to $0.45 \mathrm{~nm}$ for the $\mathrm{Si}-\mathrm{POE}_{(8)}$ treated surface, a decrease which is attributed to the filling of topographical structuring with the short $\mathrm{Si}-\mathrm{POE}_{(8)}$ leading to a smoother surface. A decrease in surface roughness after aminosilane coating of an oxidized PDMS surface has been also reported by Diaz-Quijada et al. ${ }^{19}$ In contrast, longer POE-silanes changed the topography of the surface at increased roughness values (panels $\mathrm{c}$ and $\mathrm{d}$ ) compared to oxidized PDMS (Figure 1a) yielding rms values of 0.67 and $0.98 \mathrm{~nm}$ for the $\mathrm{Si}-\mathrm{POE}_{(70)}-\mathrm{Si}$ and the $\mathrm{Si}-\mathrm{POE}_{(109)}$, respectively.

Adsorptive Attachment of POE. For our second strategy, where the adsorption of POE-containing substances to PDMS surfaces is investigated, we have chosen a triblock copolymer (trade name Pluronic F108). It consists of two 132 POE unit long tails separated by a 52 long POP unit. This triblock copolymer is known to adsorb to hydrophobic as well as to hydrophilic surfaces. ${ }^{32} \mathrm{We}$ have thus investigated the adsorption of F108 to native and oxygen plasma-treated PDMS. Table 3 demonstrates that the degree of hydrophilicity created after adsorption of F108 on plasma-treated PDMS is only slightly different compared to the two long POE-silanes. Indeed, the POE units of these three components all vary between 70 units for $\mathrm{Si}-\mathrm{POE}_{(70)}-\mathrm{Si}, 109$ units for $\mathrm{Si}-\mathrm{POE}_{(109)}$, and 132 for F108.

Contact angles of native and UV-treated PDMS on which F108 or L101 was incubated exhibit contact angles deviating by maximal $6^{\circ}$ from the contact angle of native PDMS (see Table 3) indicating that the more hydrophobic surfaces lead to a different adsorption mechanism of the Pluronic molecules. To further investigate the adsorption of the triblock copolymers onto PDMS, we have thus conducted AFM measurements of surfaces treated with F108.

To quantitatively investigate different adsorption mechanisms to hydrophilic and hydrophobic PDMS a grating mask was employed during the plasma oxidation process which allowed the patterning of hydrophobic and hydrophilic PDMS onto the same slab. In Figure 2a, an AFM image of PDMS masked with the grating during plasma exposure is shown. A cross-sectional analysis indicates that the hydrophilic PDMS surface exposed to the plasma protrudes around $(10 \pm 2) \mathrm{nm}$ compared to the unexposed and masked surface area. We attribute this to the creation of a $\left(\mathrm{SiO}_{x}\right)$ layer during the plasma process, which has been described by several authors. ${ }^{11,21,29}$ Owen et al. have proposed a layer thickness of around $10 \mathrm{~nm}$ for this silica layer. ${ }^{11}$ Interestingly, the height difference inverses after the adsorption of F108 to the plasma-treated grating (Figure 2b), so that the hydrophobic surface with adsorbed F108 now resulted in a topography-inverted image ((10 $\pm 1) \mathrm{nm}$ ). Figure 2, panels c and d, represents AFM zoom images into the hydrophilic and hydrophobic surface regions after adsorption of F108. Both surfaces are homogeneously covered; however, the hydrophobic part exhibits a rms value of $2.50 \mathrm{~nm}$ compared to a smoother coating on the hydrophilic surface $(\mathrm{rms}=0.39 \mathrm{~nm})$.

Our AFM data thus underline the recently published adsorption mechanism proposed by Barnes et al. on hydrophobic PDMS and on hydrophilic silica surfaces. ${ }^{32}$ On hydrophilic surfaces, the polymer adsorbs in a flat conformation resulting from an interaction of POE units with the hydrophilic surface. ${ }^{32,33}$ This is mainly due to H-bonding with oxygen atoms on the surface and acid

(32) Barnes, T. J.; Prestidge, C. A. Langmuir 2000, 16, 4116-21.

(33) Esipova, N. E.; Itskov, S. V.; Churaev, N. V. Colloid J. 2002, 64, $699-705$ 


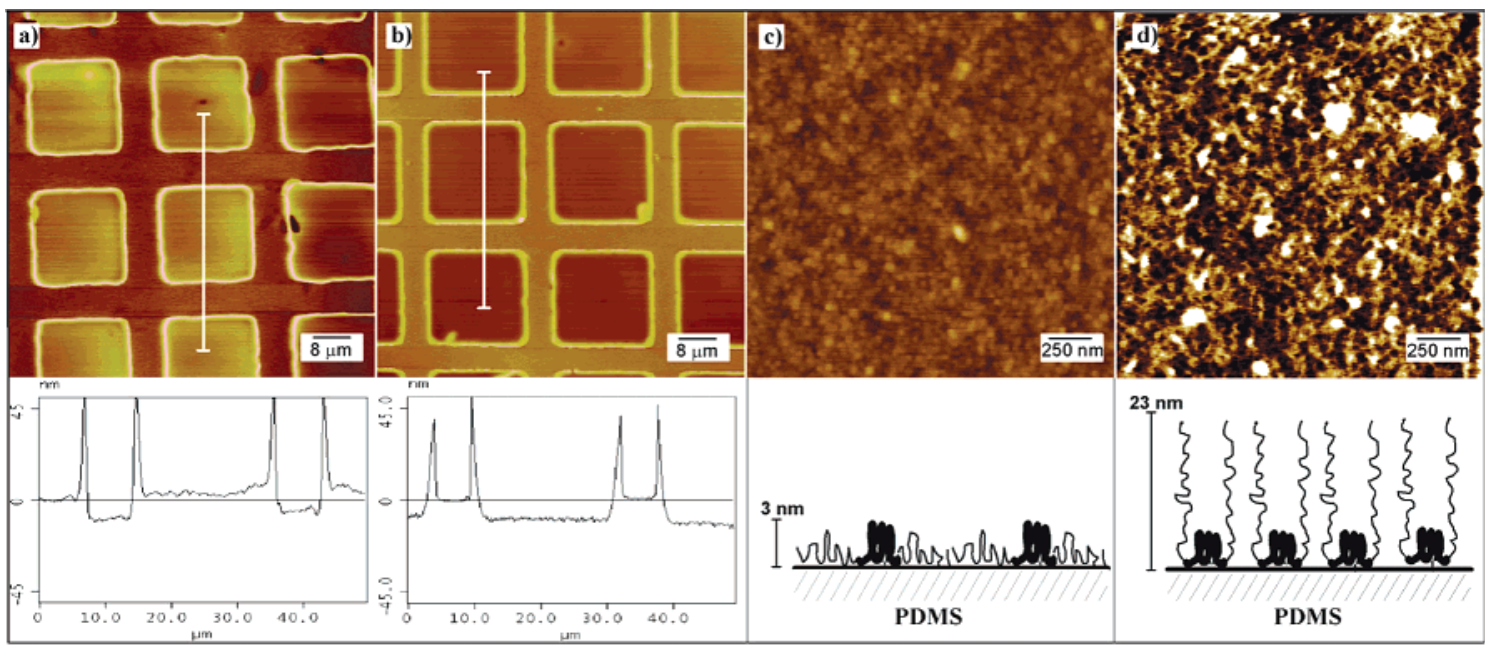

Figure 2. AFM images in phosphate buffer of (a) a native PDMS surface after plasma treatment. The surface was covered with a grating mask. The small bars represent the covered surface areas, whereas the squares were exposed to oxygen plasma. (b) The PDMS surface was exposed to an oxygen plasma with a grating mask and subsequently F108 was adsorbed. The cross-sectional analysis of the indicated lines are represented below images (a) and (b). The $z$ scale for images (a) and (b) is $100 \mathrm{~nm}$. (c) The zoom into the hydrophilic (plasma-treated) region of the grating after F108 adsorption. (d) The zoom into the hydrophobic (not treated) region of the grating after F108 adsorption. Below panels (c) and (d), drawings of the proposed molecular conformation of adsorbed F108 on hydrophilic (c) and hydrophobic (d) PDMS are schematically depicted. The $z$ scale for images (c) and (d) is $8 \mathrm{~nm}$.

base interaction, ${ }^{34}$ resulting in a flat adsorption of F108 with a reported layer thickness of $3 \mathrm{~nm} \cdot{ }^{32,35}$ On the contrary, on hydrophobic surfaces, the POP parts strongly adsorb through hydrophobic interaction extending the hydrophilic POE tail into solution..$^{32}$ The inversion of the surface profile after F108 adsorption originates from different conformations of F108 on the PDMS surface (see Figure 2, panels c and d). On hydrophobic, native PDMS, the $132 \mathrm{POE}$ unit long tail reaches into solution, forming a thick layer which is higher than the silica layer plus the $3 \mathrm{~nm}$ thick layer of F108. Adding $10 \mathrm{~nm}$ thickness for the silica layer from our AFM images to $3 \mathrm{~nm}$ layer thickness for F108 on a hydrophilic surface ${ }^{32}$ and accounting for the $10 \mathrm{~nm}$ height difference, a layer thickness of $23 \mathrm{~nm}$ for F108 on hydrophobic PDMS can be calculated. This is in reasonable agreement with a layer thickness of $20 \mathrm{~nm}$ reported by Barnes et al. ${ }^{32}$ This adsorption mechanism is additionally supported by the high roughness of F108 on hydrophobic PDMS $(\mathrm{rms}=2.50 \mathrm{~nm})$ compared to a smoother surface in the flat conformation on hydrophilic PDMS $(\mathrm{rms}=0.39 \mathrm{~nm})$. A similar adsorption mechanism for $\mathrm{F} 108$ has been reported on a hydrophilic $\mathrm{SiO}_{2}$ surface and on hydrophobic dimethylchlorosilane derivatized silicon with layer thickness of around $12 \mathrm{~nm}$ on the hydrophobic and $1 \mathrm{~nm}$ on the hydrophilic surface, respectively. ${ }^{36}$

Effect of POE Coating on Electroosmosis. The different surface coatings have been examined for their electroosmotic characteristics. Table 4 demonstrates the absolute and relative values for $\mu_{\mathrm{e}}$ in PDMS microchannels. Channels treated with the shortest POE-silane exhibit the lowest reduction of electroosmotic mobility. In the case of the longer POE units, $\mu_{\mathrm{e} 0}$ is reduced significantly in a range from 48 to $75 \%$. Careful inspection of $\mu_{\mathrm{eo}}$ reduction for the different silane components indicates an interesting trend. The smaller $\mathrm{Si}-\mathrm{POE}_{(70)}-\mathrm{Si}$ silane exhibits better EOF reduction than its longer counterpart

(34) Mathur, S.; Moudgil, B. M. J. Colloid Interface Sci. 1997, 196, 92-98.

(35) Shar, J. A.; Obey, T. M.; Cosgrove, T. Colloid Surf. A 1999, 150, $15-23$.

(36) Schröen, C. G. P. H.; Cohen Stuart, M. A.; van der Voort Maarschalk, K.; van der Padt, A.; van't Riet, K. Langmuir 1995, 11, 3068-74.
Table 4. $\mu_{\mathrm{eo}}$ of PDMS after Different Surface Treatments

\begin{tabular}{|c|c|c|c|}
\hline $\begin{array}{c}\text { surface } \\
\text { coating } \\
\text { on } \mathrm{PMDS}^{a}\end{array}$ & $\begin{array}{c}\mu_{\mathrm{eo}} \text { before } \\
\text { treatment } \\
\times 10^{-4} \\
{\left[\mathrm{~cm}^{2} / \mathrm{Vs}\right]^{b}}\end{array}$ & $\begin{array}{c}\mu_{\mathrm{eo}} \text { after } \\
\text { treatment } \\
\times 10^{-4} \\
{\left[\mathrm{~cm}^{2} / \mathrm{Vs}\right]^{a}}\end{array}$ & $\begin{array}{c}\% \text { EOF } \\
\text { reduction after } \\
\text { surface coating }\end{array}$ \\
\hline $\mathrm{Si}-\mathrm{POE}_{(8)}$ & $2.5(0.4)$ & $2.1(0.7)$ & 26 \\
\hline $\mathrm{Si}-\mathrm{POE}_{(70)}-\mathrm{Si}$ & $2.9(0.1)$ & $0.7(0.1)$ & 75 \\
\hline $\mathrm{Si}-\mathrm{POE}_{(109)}$ & $3.1(0.02)$ & $1.6(0.1)$ & 48 \\
\hline F108 (native) & $2.1(0.1)$ & $0.3(0.05)$ & 86 \\
\hline F108 (plasma) & $3.0(0.2)$ & $1.5(0.3)$ & 50 \\
\hline L101 (native) & $1.9(0.3)$ & $1.25(0.1)$ & 33 \\
\hline L101 (plasma) & $2.8(0.1)$ & $1.9(0.2)$ & 30 \\
\hline
\end{tabular}

${ }^{a}$ For silane treatment, PDMS surfaces were plasma-treated before incubation. ${ }^{b}$ The standard deviation is given in parentheses.

$\mathrm{Si}-\mathrm{POE}_{(109)}$. A stronger surface attachment through the two silane ends of $\mathrm{Si}-\mathrm{POE}_{(70)}-\mathrm{Si}$ probably combined with enhanced cross-linking could be the reason for this finding.

In the case of F108, the EOF is reduced in a more pronounced manner on the native PDMS surface than on the hydrophilic PDMS (see Table 4). The EOF reduction of F108 adsorbed to plasma-treated PDMS is in the order of the values obtained for the silane treatments $(\sim 50 \%)$. For F108 on native PDMS, the highest reduction of $86 \%$ is determined compared with all PDMS treatments. This is in reasonable agreement with the previously reported reduction to $\mu_{\mathrm{eo}}$ of $0.85 \times 10^{-4} \mathrm{~cm}^{2} / \mathrm{Vs}$ for grafted POE on PDMS. ${ }^{24}$ However, the determination of relative EOF reduction in one microchannel as demonstrated here reveals that EOF reduction is more pronounced when $\mathrm{F} 108$ adsorbs to the hydrophobic PDMS (see Table 4).

Interestingly, the Pluronic L101 with smaller POE tails shows only 30\% EOF reduction. The difference for hydrophobic and hydrophilic PDMS is marginal but reflects the trend observed for F108 (Table 4). It could thus be demonstrated that both for POE-silanes as well as Pluronic components the EOF reduction after coating is $\mathrm{POE}$ chain-length dependent; the longer the POE tail the higher the EOF reduction.

It is commonly argued that anchoring polymer tails in the double layer of charged surfaces can significantly reduce $\mu_{\mathrm{eo}}$ due to an increase in viscosity. ${ }^{37}$ We assume that F108 on native and hydrophobic PDMS extends through the complete double layer and in consequence 

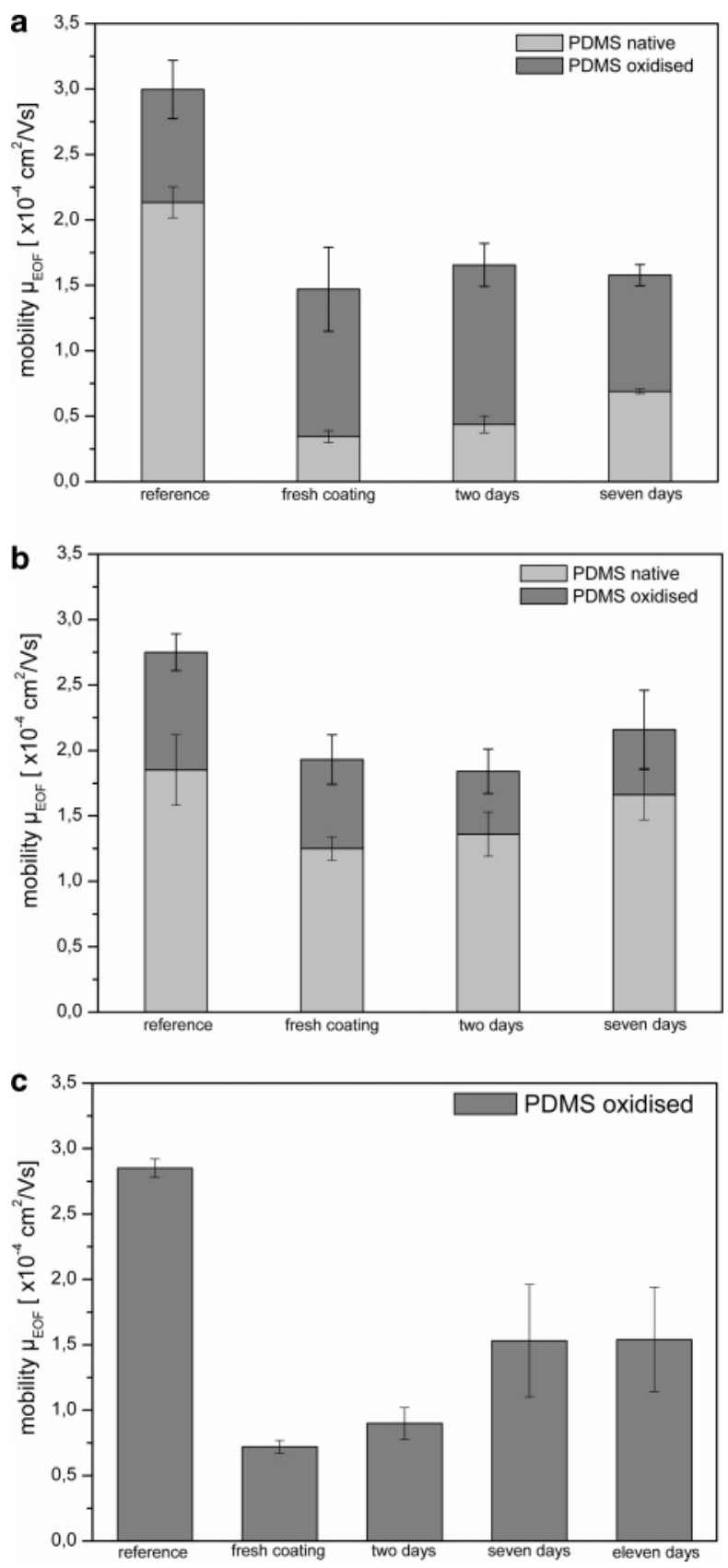

Figure 3. Long-term stability of POE derivatives on PDMS probed by EOF measurements: (a) EOF values for periods of 7 days on hydrophobic (light gray) and hydrophilic (dark gray) PDMS. The first bar represents the reference measurement before the coating. (b) L101 coatings probed for 7 days as in (a). (c) EOF values for periods of 11 days for oxidized PDMS channels coated with $\mathrm{Si}-\mathrm{POE}_{(70)}-\mathrm{Si}$.

increases the viscosity more effectively, thus having a higher impact in EOF reduction. This picture reflects the data obtained by AFM imaging, yielding a $23 \mathrm{~nm}$ layer for F108 on native PDMS compared to $\sim 3 \mathrm{~nm}$ on hydrophilic surfaces, ${ }^{32}$ such as plasma-treated PDMS. Additionally, the overall charge on the hydrophobic surface is smaller than on the hydrophilic PDMS which reduces the zetapotential and thus the $\mu_{\mathrm{e} o}$.

Finally, the long-term stability of EOF in PDMS microchannels coated with F108, L101, and $\mathrm{Si}-\mathrm{POE}_{(70)}-$ $\mathrm{Si}$ has been investigated. Figure 3 demonstrates the resulting $\mu_{\mathrm{eo}}$ over the period of several days. After 7 days

(37) Grossman, P. D.; Colburn, J. C. Capillary Electrophoresis, Theory and Practice; Academic Press: San Diego, CA, 1992. of storage, the $\mu_{\mathrm{eo}}$ of a hydrophilic PDMS channel treated with F108 resulted in $(1.6 \pm 0.1) \times 10^{-4} \mathrm{~cm}^{2} / \mathrm{Vs}$, which is practically the initially measured $\mu_{\text {eo }}$ value $((1.5 \pm 0.3) \times$ $10^{-4} \mathrm{~cm}^{2} / \mathrm{Vs}$ ) on a freshly coated surface. On the hydrophilic surface, the $\mu_{\mathrm{e}}$ is stable within the error of the experiment. Although no continuous flushing with buffer solution was performed, this channel was submitted for a minimum of $2 \mathrm{~h}$ to an electric field of $250 \mathrm{~V} / \mathrm{cm}$ for each EOF measurement so that the buffer content was flushed extensively through these channels. Thus, the stable longterm mobility indicated a stable surface coating created by an adsorptive treatment with a triblock copolymer. On the hydrophobic PDMS, the EOF reduction is more pronounced; however, the $\mu_{\mathrm{eo}}$ shows a tendency to increase after one week, thus indicating a less stable coating (see Figure 3a).

PDMS surfaces treated with L101 show a similar behavior. EOF on native PDMS increases slightly with time, whereas it remains constant for the oxidized surfaces (Figure $3 \mathrm{~b}$ ). The adsorption of triblock copolymers on hydrophobic surfaces has been tested for protein separations in capillary electrophoresis applications and revealed excellent separation performances for over 80 runs. ${ }^{14}$ It can thus be expected that the presented surface coating is also applicable to electrophoretic measurements in PDMS microchannels.

The long-term stability of $\mathrm{Si}-\mathrm{POE}_{(70)}-\mathrm{Si}$ coated oxidized PDMS surfaces is demonstrated in Figure 3c. Interestingly, $\mu_{\mathrm{e}}$ increases from $0.72 \times 10^{-4} \mathrm{~cm}^{2} / \mathrm{Vs}$ for the fresh coating to a value of $1.53 \times 10^{-4} \mathrm{~cm}^{2} / \mathrm{Vs}$ after one week and levels off for more extended times. We attribute this to a detachment of only weakly adsorbed POE-silane molecules.

\section{Conclusion}

Covalent attachment mediated by alkoxysilanes as well as adsorptive attachment have been demonstrated to effectively coat PDMS surfaces with POEs in microchannels. In particular, contact angle measurements demonstrated better attachment on oxygen plasma-treated surfaces than on native and UV-treated surfaces of the alkoxysilane due to a more pronounced creation of silanol groups on the plasma-treated surfaces. AFM images revealed good surface coverage for all three alkoxysilanes.

Adsorption of F108 has been demonstrated on hydrophobic (native) as well as on hydrophilic (oxygen plasmatreated) surfaces. AFM studies support adsorption mechanisms similar to those reported previously. ${ }^{32}$ On the hydrophilic surface, F108 adsorbs in a flat conformation, and adsorption is mediated through hydrogen bonding of POE tails to the surface. On hydrophobic surfaces, the hydrophobic POP part of F108 attaches to the surface. As a consequence, the hydrophilic $\mathrm{POE}$ part reaches into solution, creating a thicker POE layer.

Electroosmotic mobility studies revealed that all surface coatings reduced EOF. However, the molecules with the smaller POE tails, Si-POE $(8)$ and L101, had the lowest impact. A more pronounced effect on the viscosity near the PDMS buffer interface was only established for the longer POE tails (POE units $>70$ ) with the consequence of lower $\mu_{\mathrm{eo}}$. Tailoring POE length could thus be an instrument to control $\mu_{\mathrm{eo}}$ in PDMS microchannels.

In comparison to a previously published strategy of attaching silanes to control EOF in PDMS devices, ${ }^{20,38}$ our method is carried out in an aqueous environment so that unwanted swelling of PDMS or adsorption of organic

(38) Wang, B.; Abdulali-Kanji, Z.; Dodwell, E.; Horton, J. H.; Oleschuk, R. D. Electrophoresis 2003, 24, 1442-50. 
solvent molecules is prevented. The simple adsorption route with F108 is even more easily implemented in the pre-preparation process of PDMS microfluidic devices due to the fact that the adsorption can easily be carried out before a separation experiment. Furthermore, no additional irradiation equipment is necessary as in the case of UV mediated graft polymerization of POE on PDMS channels. $^{24}$

Long-term stability and the behavior of these coatings under injection and separation conditions have to be investigated further. However, due to the fact that longterm stability of POE coatings has been reported for capillary electrophoresis, ${ }^{14}$ their use in PDMS microfluidic channels appears very promising. Also, the good resistance to protein adsorption of POE coated surfaces ${ }^{13}$ during electrophoresis makes these coatings especially interesting for electrophoretic separations of proteins in PDMS channels.

Acknowledgment. Financial support from the Deutsche Forschungsgemeinschaft (Project Number An 370/ 1-1 and 2) and generous Pluronics sample donation by BASF (Dr. Stephan Altmann and Dr. Kati Schmidt) are gratefully acknowledged. Sincere thanks are given to Dr. Armin Brechling and Prof. Ulrich Heinzmann (Physics of Molecules and Surfaces, Bielefeld University) for technical assistance in contact angle measurements. We acknowledge Nicole Griemla for assistance in performing some AFM characterization and Kay Lofthouse for proof reading the manuscript.

LA0510432 